\title{
Assistance au suicide: surveiller tout en donnant sa place à l'éthique
}

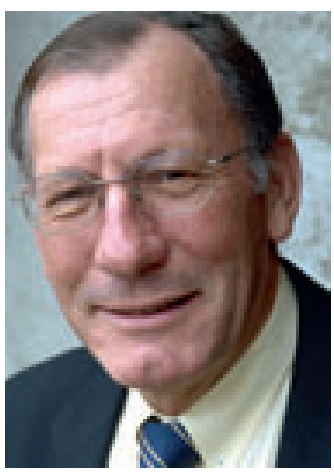

Jean Martin
L'Académie suisse des sciences médicales (ASSM) a fait connaître son opposition aux propositions du Conseil fédéral pour une modification de l'art. 115 du Code pénal [1]. Ses arguments sont fort pertinents, en particulier quand elle relève qu'une surveillance qui pourrait prendre des aspects chicaniers - de la pratique de l'assistance au suicide (AS) organisée ferait que les médecins praticiens seront plus sollicités à titre individuel.

Qu'on me permette de différer de l'Académie sur sa critique selon quoi l'AS serait «institutionnalisée en tant qu'activité médicale». Le fait qu'elle ne soit pas partie du «noyau dur» de l'activité du médecin est incontestable; mais, pour suivre la problématique de longue date, j'en suis venu à me demander si on ne devait pas, pragmatiquement, admettre ce que connaissent trois Etats américains, dont l'Oregon, et en Europe les pays du Benelux, à savoir la notion de physician assisted suicide. Ce n'était pas l'idée initiale du Code pénal mais, dans le passé récent, c'est la réalité de ce qui existe chez nous dans des situations de patients gravement malades. De plus, l'intention du Conseil fédéral d'exiger que le moyen employé soit soumis à prescription médicale apparaît raisonnable. Une responsabilité médicale est, qu'on le veuille ou non, partie intégrante du processus - la clause de conscience du médecin étant toujours réservée.

Cette responsabilité (assumée à titre personnel et en son âme et conscience) comporte la prescription du produit. D'un point de vue déontologique et de bonne pratique, il devrait aller sans dire qu'elle inclut aussi le fait de s'assurer de la capacité de discernement de la personne (constatation de bon sens sans qu'il soit besoin d'expertise), ainsi que la prise de connaissance du dossier démontrant que cette personne est gravement malade ou handicapée, que sa qualité de vie est devenue très mauvaise, qu'elle est continuellement dépendante et souffre, que la situation est irréversible et que les mesures envisageables de soulagement ou accompagnement ont été proposées et discutées. NB: c'est dire entre autres, comme le fait l'Académie, que la condition expresse de «mort imminente» doit être écartée.

A mon sens, il faut éviter absolument de diluer les responsabilités des personnes impliquées. Sans contestation possible, il y a deux acteurs principaux: le patient exprimant de manière répétée sa demande et le médecin qui prescrit une dose létale de pento- barbital. Pour avoir une pratique éthiquement solide, il faut mettre l'accent sur la responsabilité de ces acteurs. Contrairement au modèle indûment complexe du Conseil fédéral, l'implication d'un seul médecin est adéquate. Si au contraire on introduit diverses vérifications supplémentaires (au risque qu'elles deviennent administrativement banalisées), on déresponsabilise ce médecin. En général, on ne doit pas craindre de mettre l'accent sur l'éthique et la déontologie du praticien [2], dont c'est le métier d'avoir à prendre des décisions qui mettent en jeu la vie et la mort (y compris dans des euthanasies directes qui restent confidentielles mais existent).

Un autre point à souligner est que le statu quo au plan de la loi - n'est pas la plus mauvaise solution. A la place d'articles nouveaux, il s'agirait de faire fonctionner au mieux les clauses générales de surveillance/ police au niveau cantonal. Ainsi dans le champ médico-sanitaire par les Départements chargés de la santé, qui peuvent intervenir chaque fois qu'une préoccupation surgit, par exemple en rapport avec le comportement de professionnels. S'agissant de considérations d'ordre public, but lucratif éventuel, etc., d'autres Départements cantonaux et/ou les Ministères publics ont des compétences. Rappelons aussi que tout suicide est une mort non-naturelle qui exige l'appel au Juge d'instruction; il y a là un contrôle systématique de valeur même s'il est a posteriori. Dans ce modèle «statu quo légal», la surveillance est, c'est vrai, générale, non précisée dans les textes mais elle est réelle, y compris par le «contrôle social» qui reste une réalité dans les entités que sont les cantons (une longue expérience me l'a montré, nous devons tirer avantage de ce qu'autorise notre petitesse). Et on évite le foisonnement bureaucratique dont on peut craindre le développement, avec des risques pour la protection de données personnelles sensibles.

L'ASSM «considère que ses directives constituent un outil adéquat». Avec elles et les rapports de la Commission nationale d'éthique, on dispose effectivement de documents capables d'orienter l'action de contrôle des services publics, y compris des tribunaux; rappelons qu'on parle à cet égard de bases paralégales (soft law - voir aussi [2]), qui ont toute leur utilité en l'absence de loi spécifique - et sont plus proches de la réalité de la vie des gens qu'un article de loi.

Jean Martin* médecin cantonal vaudois et membre de la rédaction du BMS. 